\title{
EFFICIENT IMPLEMENTATION OF WIDEBAND MULTIBEAM FORMING NETWORK USING SOPOT COEFFICIENTS AND MULTIPLIER BLOCK
}

\author{
S.C. Chan, Carson K.S. Pun and K. L. Ho \\ Department of Electrical and Electronic Engineering \\ The University of Hong Kong, Pokfulam Road, Hong Kong \\ scchan@eee.hku.hk, kspun@eee.hku.hk,klho@eee.hku.hk
}

\begin{abstract}
This paper proposes an efficient implementation structure for the wideband multi-beam forming network in beamspace adaptive array for wideband signals. The filter coefficients of the beamforming network are represented as sum-of-powers-to-two coefficients (SOPOT) and are implemented using the multiplier block technique. The SOPOT representation allows the coefficients to be implemented as limited number of adders and shifters without any multiplications. The redundancy in the SOPOT coefficients are further removed by the multiplier block technique to yield minimum adder realization. Design results showed that significant reduction in hardware complexity can be obtained by the proposed structure as compared with conventional direct form implementation.
\end{abstract}

\section{INTRODUCTION}

Wideband beamforming using sensor arrays is an effective method for suppressing interference whose angles of arrival are different from the desired looking direction. They find important applications in radio communications, sonar, radar, and acoustics [1-3]. Traditional adaptive wideband beamformer usually employs tapped-delay line with adaptive coefficients to generate appropriate beam patterns for interference suppression. This usually requires considerable number of adaptive coefficients resulting in rather long convergence time and high implementation complexity. Several methods including the use of adaptive infinite impulse response filter (IIR) [12] and beamspace adaptive arrays [4,5] were proposed to overcome these problems. For the beamspace adaptive arrays in [5], interference suppression is achieved by combining the outputs from a fixed beamforming network which generates a set of beams pointing in different directions (Fig. 1). Since the number of beams selected is usually small, the number of adaptive weights for combining them are greatly reduced, resulting in faster convergence and fewer number of high speed variable multipliers. Although the number of variable multiplier is significantly reduced, the fixed beamforming network does require large number of fixed multipliers. Moreover, the complexity increases with the number of antenna, number of beams and the spatial-temporal selectivity of the fan filters in the beamforming network. In this paper, a novel implementation of the fixed beamforming network using canonical signed digits (CSD) representation (or sum-of-powers-of-two coefficients (SOPOT) ) of the filter coefficients and a technique called multiplier block (MB) [11] is introduced. More precisely, the coefficients of the beamforming network are represented in CSD which requires only limited number of additions and hardwired shifts for implementation. In addition, the number of additions to realize multiplications with the CSD coefficients can further be reduced by implementing the multiplications as a multiplier block. Multiplier block is an efficient method for performing the multiplications of a multiplcand with a set of fixed constant multipliers, by sharing subexpressions or immediate results common to the multipliers. In principle, it is possible to remove the redundancies in the constant multiplications leading to a minimum-adder-graph [11] with minimum adder realization. Design results show that the implementation complexity of the fixed beamforming network can be reduced drastically by the proposed technique. For example, with $N$ sensors and $L$ beams in the multibeam forming network each with a length- $M$ FIR filter, the total number of multipliers required is $L M N$. For $L=5, N=15, M=15$, direct implementation will require $L \cdot M \cdot N=1125$ multipliers. On the other hand, if the coefficients are represented as SOPOT coefficients with 8 -bit accuracy, then the proposed structure requires only 53 adders with an admirable frequency response. It should be noted that the proposed method is also applicable to the frequency invariance beamformer (FIB) in [6,7] and the infinite impulse response (IIR) multibeam former in [8][9], which approximates the fixed finite impulse response (FIR) filters in the multibeam former network by IIR filters. The complexity is reduced by a factor of $56.9 \%$ for $L=12, N=31, M=51$. However, the implementation of the IIR filters is somewhat complicated due to their finite wordlength effect. In addition, the redundancy in the filter coefficients are not fully explored and the overall complexity still grows rapidly with the number of taps, beams and antennas in the multibeam network. Although the multiplier block technique can also be used to realize efficiently these IIR filters, we only consider the FIR case because of its simplicity mentioned above and the large amount of redundancy existing in the coefficients as we shall see later in Section IV.

This paper is organized as follows: in section II, the principle of the beamspace adaptive beamformer and the design of the fixed beamforming network are described. Section III is devoted to the proposed implementation of the beamforming network. This is followed by the design results in Section IV. Finally, conclusions are drawn in section V.

\section{BEAMSPACE ADAPTIVE BEAMFORMER USING} FAN Filter

Fig. 1 shows the structure of the beamspace adaptive array for wideband signals proposed in [5]. The digital received signal received from the sensors, after analogue-to-digital conversion and possibly downconversion, are fed into a wideband multibeam forming network, which is a set of spatial filters with fixed directions and hence coefficients. If $x_{k}(n), k=0, \ldots, N-1$, is the received signal from the $k^{\text {th }}$ sensor, the output of the $l^{\text {th }}$ beam forming network is

$$
y_{l}(n)=\sum_{k=0}^{N} \sum_{m=0}^{M-1} h_{l, k}(m) x_{k}(n-m),
$$

where $h_{l, k}(m)$ is the impulse response of the $l^{t h}$ beamforming network. Its frequency response [5] as a function of $f$ and $\theta$ is given by

$$
\begin{aligned}
& H_{l}(f, \theta)=\sum_{k=0}^{N-1} \sum_{m=0}^{M-1} h_{l, k}(m) \cdot e^{-j 2 \pi m m T_{s}} \cdot e^{j 2 \pi f(d \sin \theta / c) k}, \\
& l=1,2, \cdots L
\end{aligned}
$$

where $T_{s}$ is the sampling period, $f$ is the frequency, $\theta$ is the angle of arrival (AOA) or direction of arrival (DOA) measured from the normal of the linear array, $d$ is the antenna spacing and $c$ is the propagation speed of the receiving signal. The 
number of sensors is $N$ and the number of taps per beam is $M$. By introducing the frequency variables $f_{1}$ and $f_{2}$ as follows

$$
f_{1}=f \cdot T_{s} \text { and } f_{2}=f \cdot d \sin (\theta) / c \text {, }
$$

(2) can be rewritten in the familiar form of a $2 \mathrm{D}$ digital filter

$$
H_{l}(f, \theta)=\hat{H}_{l}\left(f_{\mathrm{l}}, f_{2}\right)=\sum_{k=0}^{N-1} \sum_{m=0}^{M-1} h_{l, k}(m) \cdot e^{-j 2 \pi n f_{1}} \cdot e^{j 2 \pi k f_{2}} .
$$

To approximate a flat unit response for all frequencies $f$ at a fixed direction $\theta_{t}$ (either for receiving the desired signal or suppressing the interference by subtracting it from the main beam), the ideal response of $H_{l}(f, \theta)$, up to a certain delay, should be

$$
H_{l}\left(f, \theta_{l}\right)=\delta\left(\theta-\theta_{1}\right)=\hat{H}_{1}\left(f_{1}, f_{2}\right) \text {, }
$$

where $\delta(\theta)$ is the delta function. (5) suggests that the ideal response of $H_{l}\left(f, \theta_{l}\right)$ in the $\left(f_{1}, f_{2}\right)$-plane, i.e. $\hat{H}_{l}\left(f_{1}, f_{2}\right)$, should be a line with unit amplitude as given by

$$
f_{2}=f_{1} \cdot d \sin \left(\theta_{l}\right) /\left(c T_{s}\right) \text {, }
$$

according to (3). In practice, the array spacing of a linear array $d$ is usually chosen as $\lambda / 2$ to avoid undersampling of the receiving signal and too much coupling between the antennas. Fig. 2 shows such a line at angle $\theta_{t}$ with $d=\lambda / 2=c T_{s}$. In practice, $H_{l}\left(f, \theta_{i}\right)$ is approximated as a set of fan filters with passband given by the shaded area in Fig. 2. The outputs from the $L$ beams of the multibeam forming network, which represents received signal in directions $\theta_{l}, l=1, \ldots, L$, are adaptively selected and combined using the LMS algorithm to form an estimate of the interfering signals to be subtracted from the main beam (beam 0 with $\theta_{0}=0^{\circ}$ is assumed to be the looking direction). The coefficients of the fan filter can be determined by the transformaton and windowing method proposed in [5], which is also used in this work. More precisely, a one dimension prototype filter with desired bandpass response and zeros at the center frequencies of other beams is first designed. It is then transformed by means of the transformation to obtain the frequency response of the fan filter. The frequency response of the fan filter in the grey area of Fig. 2 is set to zero, though it can be chosen arbitrary. An inverse discrete fourier transform is then applied to the sampled frequency response to obtain the desired filter coefficients. This 2-D impulse response is then windowed to obtain the final filter coefficients.

\section{NOVEL IMPLEMENTATION OF THE MULTIBEAM FORMING NETWORK}

It can be seen from (1) that the beam forming network for the $l^{\text {th }}$ beam is actually obtained by summing the outputs of a set of FIR filters, each with its input coming from one sensor element in the sensor array. To see this, rewrite (1) as follows

$$
y_{l}(n)=\sum_{k=0}^{N-1} \sum_{m=0}^{M-1} h_{l, k}(m) x_{k}(n-m)=\sum_{k=0}^{N-1} g_{l, k}(n)
$$

where $g_{l, k}(n)=\sum_{m=0}^{M-1} h_{l, k}(m) x_{k}(n-m)$ is the output of the FIR filter with impulse response $h_{l, k}(m)$ and its input is derived from the $k^{\text {th }}$ sensor element (see Fig. 3 for the overall structure). To achieve a multiplier-less realization of the multibeam forming network, let's replace the coefficients $h_{i, k}(m)$ in the FIR filters as the following sum of powers-of-two coefficients (SOPOT) or canonical signed digits (CSD), $b=\sum_{k=0}^{L-1} a_{k} \cdot 2^{-b_{k}}$, where $a_{k}$ is either 1 or -1 , and $b_{k} \in\left\{-l_{L}, \ldots .1,0, \ldots l_{U}\right\}$. The larger the numbers $l_{L}, l_{U}$, and $L$, the closer the SOPOT approximation will be to the original real number. In practice, the number of non-zero terms is usually kept to a small number while satisfying a given specification so that the multiplication can be implemented by a limited number of shift and add (subtract) operations, giving rise to multiplierless realization. As mentioned earlier, the number of adders required to implement $h_{l, k}(m)$ in SOPOT representation can further be reduced using the technique of multiplier block. The idea is to rewrite $h_{l, k}(m)$ in transposed form for computing $g_{l, k}(n)=\sum_{m=0}^{K} h_{l, k}(m) x_{k}(n-m)$ as shown in Fig. 3. It can be seen that instead of multiplying the delayed input samples with the filter coefficients as in the direct form, the input sample is now multiplied with all the coefficients. This can be efficiently implemented using a multiplier block (MB) [9]. Let's consider a simple example with two filter coefficients: 3 and 21 . The SOPOT representations of these two numbers are: $3=2^{1}+1$ and $21=2^{4}+2^{2}+1$. This requires 3 adders and 3 shifts. If implemented in a $\mathrm{MB}$, the multiplication of the input with the coefficient 3 will also be generated by decomposing 3 as $2^{1}+1$, requiring one addition. The multiplication with 21 , however, can be simplified by re-using the intermediate result generated by the first filter coefficient ' 3 ' as $21=3 \cdot 7=3 \cdot\left(2^{3}-1\right)$. Actually, the intermediate result, after multiplication by 3 , is multiplied by 7 , which requires one less adder than generating 21 directly. In principle, it is possible to remove all the redundancy found in the constant multipliers leading to a realization with the minimum number of adders. This can drastically reduce the number of adders required for realizing the multibeam forming network, as will be illustrated later in Section IV. A further saving can be obtained by grouping $h_{l, k}(m)$ with a fixed value of $k$ and implement them as one multiplier block (MB\#0, to MB\#N-1 in Fig. 3). Therefore all the redundancy in $h_{i, k}(m)$, $l=1, \ldots, L ; m=0, \ldots, M-1$, for a fixed value of $k$ can be removed, yielding a minimum adder realization. The generation of the MB follows the method of Dempster et al. proposed in [11]. Interested readers are referred to [11] for more details. Next, we consider a design example.

\section{DESIGN EXAMPLE}

For comparison, we consider the same example designed in [5] The number of antenna elements is 15 . All the digital FIR filters has 15 taps. The multibeam forming network consists of 5 beams pointing at angles $0^{\circ}, \pm 24^{\circ}$ and $\pm 53^{\circ}$, respectively. Figure 4 shows their frequency responses when the filter coefficients are represented in CSD with 8-bit accurarcy (as the frequency responses at the angles $\pm 53^{\circ}$ and $\pm 24^{\circ}$ are symmetric, only the beam patterns at $-53^{\circ},-24^{\circ}$ and $0^{\circ}$ are shown). Due to page limitation, the frequency responses with different wordlength for the filter coefficients are omitted here. It was found that the 8-bit SOPOT frequency response is comparable even with the full precision results. Table 1 compares the hardware complexity of using the conventional method, the proposed SOPOT coefficient method, and the proposed SOPOT-MB method in implementing the multibeam forming network under consideration. The conventional method requires 1125 multipliers. (excluding the 1050 additions for implementing the delay line additions required in the FIR filters) The proposed SOPOT realization reduces the implementation complexity to 350 adders for 8-bit accuracy and 3140 adders for 16-bit accuracy. Using the multiplier block technique, the number of adders goes down to 53 for 8-bit accurary and 922 for 16-bit accuracy. An order of magnitude improvement in hardware complexity is achieved by the proposed method. It can therefore be concluded that the proposed method can 
drastically reduce the hardware complexity in implementing the multibeam forming network for the beamspace wideband adaptive beamformer.

\section{CONCLUSION}

A novel and hardware efficient structure for implementing the wideband multibeam network for beamspace adaptive array is presented. The proposed struture, which is based on the SOPOT representation of the filter coefficients and the multiplier block technique, significantly reduces the hardware complexity compared with conventional direct form implementation.

\section{REFERENCES}

[1] D. H. Johnson and Dan E. Dudgeon, Array signal processing : concepts and techniques, Englewood Cliffs, NJ : Prentice Hall, 1993.

[2] H. Krim and M. Viberg, "Two decades of array signal processing research: the parametric approach," IEEE Signal Processing Mag., vol. 13, pp. 67-94, Jul. 1996

[3] B. D. Van Veen and K. M. Buckley, "Beamforming: a versatile approach to spatial filtering," IEEE ASSP Mag., vol. 52, pp. 4-24, Apr. 1988.

[4] K. Nishikawa, T. Yamamoto, K. Oto and T. Kanamori, "Wideband beamforming using fan filter," in Proc. IEEE ISCAS'92, vol. 2. pp. $533-536,1992$.

[5] T. Sekiguchi and Y. Karasawa, "Wideband beamspace adaptive array utilizing FIR Fan filters for multibeam forming," IEEE Trans. Signal Processing, vol. 48, pp. 277-284, Jan. 2000.

[6] D. B. Ward, R. A. Kennedy and R. C. Williamson, "Theory and design of broadband sensor arrays with frequency invariant farfield beam patterns." J. Acoust. Soc. Amer., vol. 97, no.2, pp.1023 1034, Feb. 1995

[7] D. B. Ward, R. A. Kennedy and R. C. Williamson, "FIR filter design for frequency invariant beamformers," IEEE Signal Processing Lett., vol.3, pp. 69-71, Mar. 2000.

[8] M. Ghavami and R. Kohno, "Recursive fan filters for a broad-band partially adaptive antenna," IEEE Trans. Commun., vol. 48, pp. 185-188, Feb. 2000

[9] S. Forcellini and R. Kohno, "Frequency invariant beamformer using a single set of IIR filter coefficients and multirate techniques," in Proc. IEEE $6^{\text {th }}$ International Symposium on Spread-Spectrum Technique and Application, New Jersey, USA vol. 2, pp.575-579, Sept.6-8, 2000

[10] L. J. Griffiths and C. W. Jim, "An alternative approach to linearly constrained adaptive beamforming," IEEE Trans. Antennas Propagat., vol. AP-30, pp. 27-34, Jan. 1982.

[11] A. G. Dempster and M. D. Macleod, "Use of minimum-adder multiplier blocks in FIR digital filters," IEEE Trans. Circuits and Syst. II, vol. 42, no. 9, pp. 569-577, Sep. 1995.

[12] C. N. Adkins and J. J. Turtora, "A broadband beam former with pole-zero unconstrained jammed rejection in linear arrays," IEEE Trans. Signal Processing, vol. 44, pp.438-441, Feb.1996

\begin{tabular}{|c|c|c|}
\hline & \multicolumn{2}{|c|}{ Direct implementation } \\
\hline No. of mul. & \multicolumn{2}{|c|}{$15 \times 15 \times 5=1125$} \\
\hline & \multicolumn{2}{|c|}{ No. of Adders } \\
\hline $\begin{array}{l}\text { No. of bits/ } \\
\text { coefficient }\end{array}$ & $\begin{array}{c}\text { Proposed structure } \\
\text { SOPOT only }\end{array}$ & $\begin{array}{c}\text { Proposed structure } \\
\text { SOPOT and MB }\end{array}$ \\
\hline 8 & 350 & 53 \\
\hline 9 & 582 & 88 \\
\hline 10 & 1001 & 146 \\
\hline 11 & 1297 & 234 \\
\hline 12 & 1703 & 361 \\
\hline 13 & 2033 & 487 \\
\hline 14 & 2410 & 668 \\
\hline 15 & 2772 & 792 \\
\hline 16 & 3140 & 922 \\
\hline
\end{tabular}

Table 1. Hardware complexity of various methods. $N=15$, $M=15, L=5$. (Only consider all the FIR filters in the BFNs, excluding the delay line addition.)

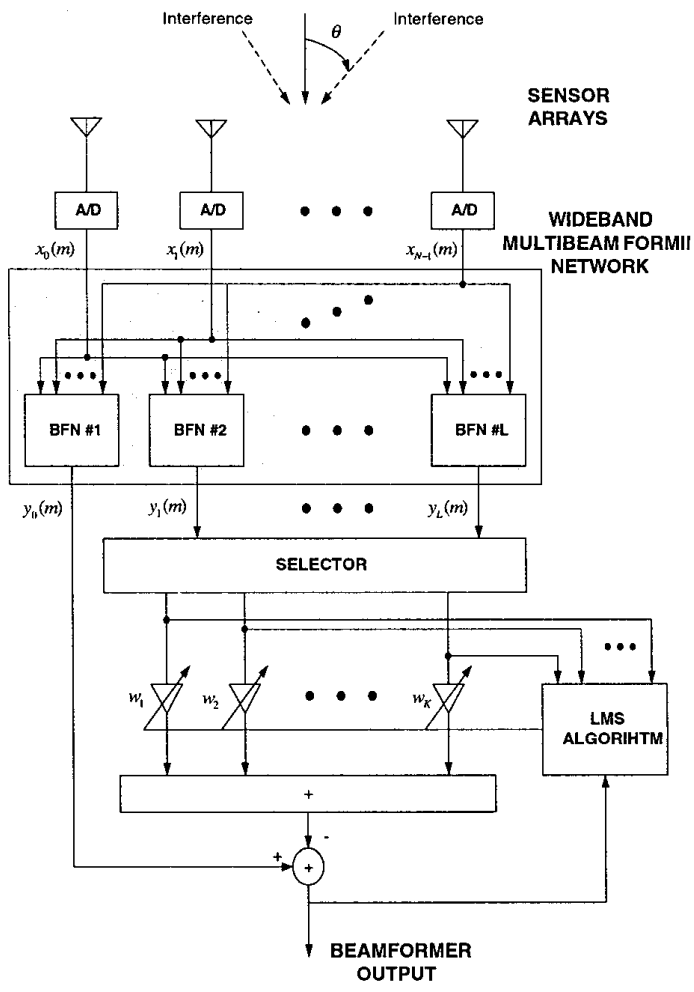

Figure 1. Beamspace adaptive array for wideband signals. Beamforming network (BFN).

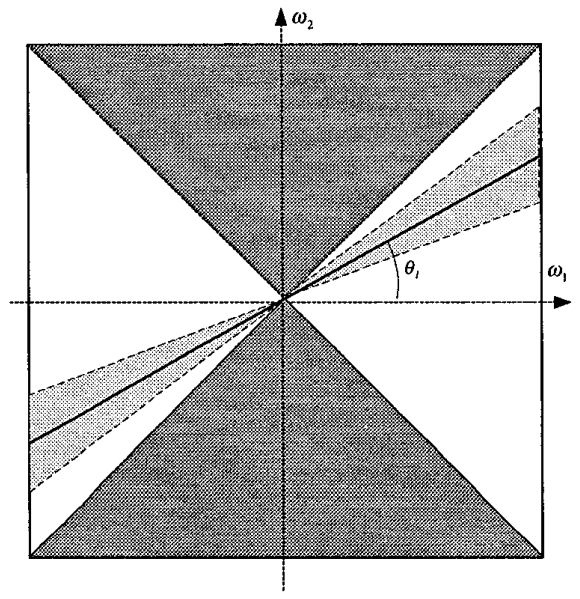

Figure 2. Frequency response of the ideal fan filter. 


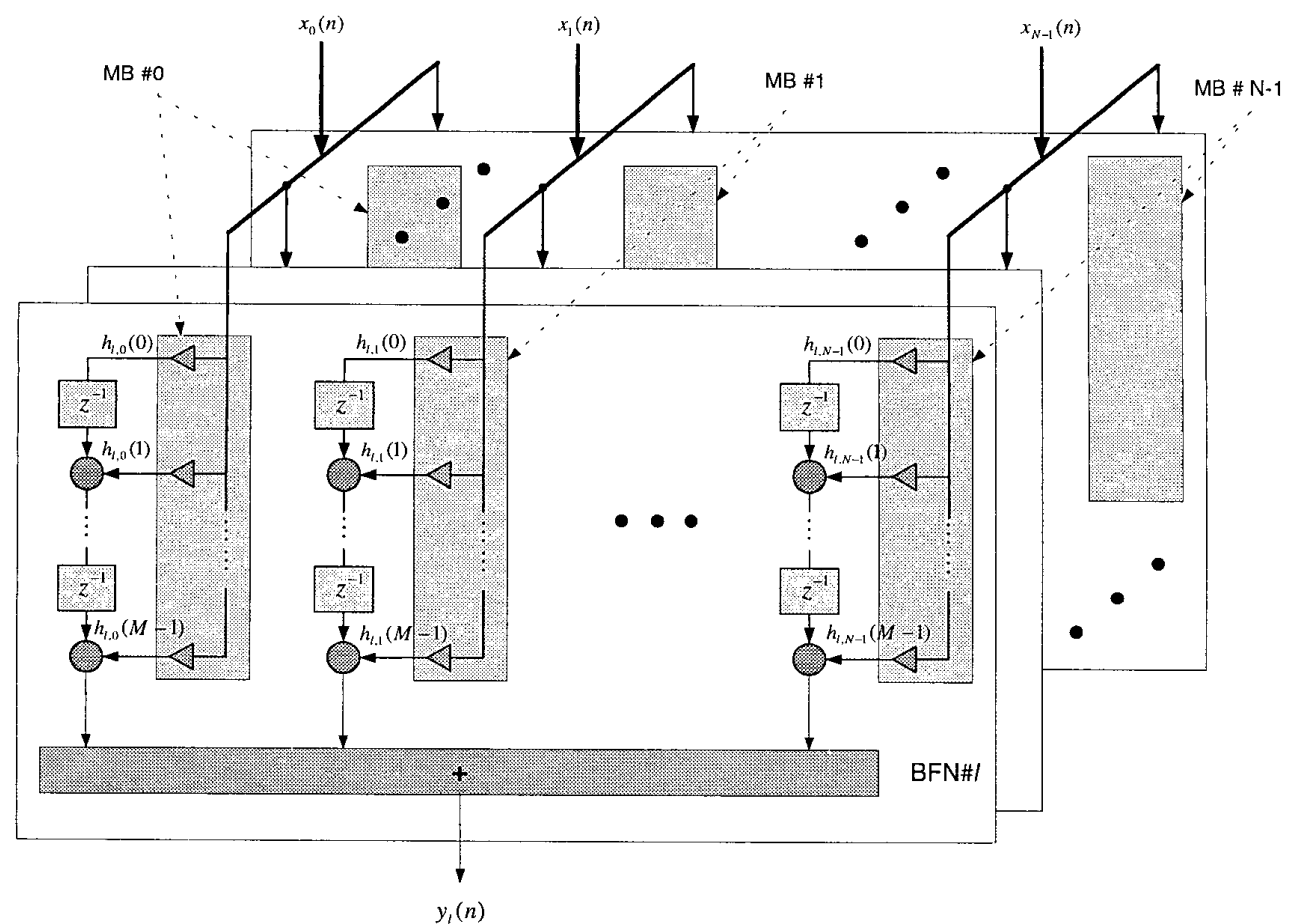

Figure 3. Proposed multibeam forming network using SOPOT coefficients and multiplier block (MB).
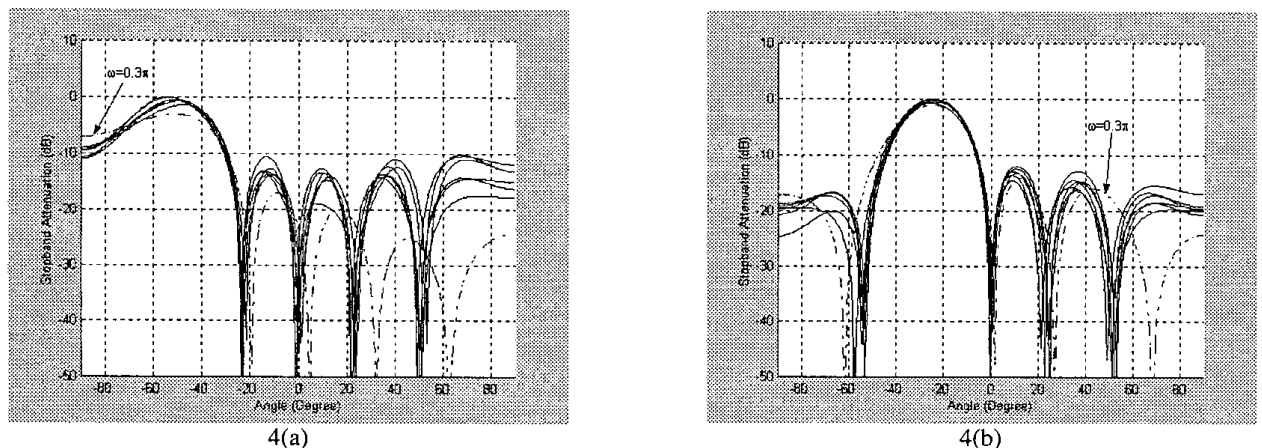

4(a)

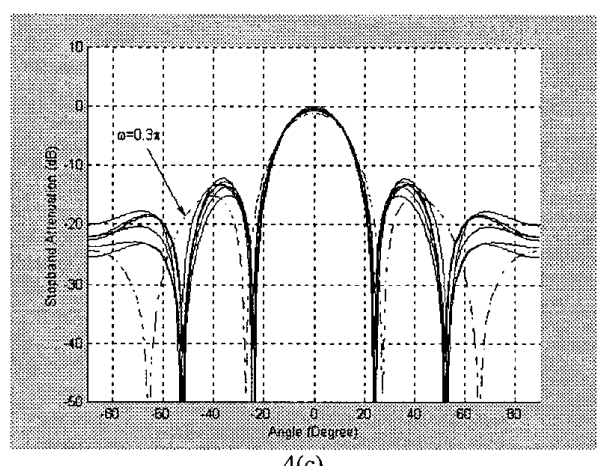

4(b)

$4(c)$

Figure 4. Frequency response of the multibeam forming network at seven frequencies: $0.3 \pi, \cdots, 0.9 \pi$ (a) $-53^{0}$, (b) $-24^{0}$, (c) $0^{0}$. 